\title{
On nonoscillatory solutions tending to zero of third-order nonlinear dynamic equations on time scales
}

\section{Yang-Cong Qiu*}

\section{"Correspondence:} q840410@qq.com

School of Humanities and Social

Science, Shunde Polytechnic

Foshan, Guangdong 528333,

P.R. China

\section{Springer}

\begin{abstract}
By Kranoselskii's fixed point theorem, we state some sufficient conditions of the existence of nonoscillatory solutions tending to zero as $t \rightarrow \infty$ of a class of third-order nonlinear dynamic equations on time scales. Two examples are presented to show the significance of the results.
\end{abstract}

MSC: 34N05; 34C10;39A13

Keywords: third-order nonlinear dynamic equations; time scales; existence; nonoscillatory solutions tending to zero; Kranoselskii's fixed point theorem

\section{Introduction}

In 1988, Hilger introduced the theory of time scales in his Ph.D. thesis [1]; see also [2]. We refer the reader to $[3,4]$ for details on time scales. We remark that some researchers have studied the existence of nonoscillatory solutions of several kinds of nonlinear dynamic equations on time scales, which can be found in [5-10].

Zhu and Wang [10] studied first-order nonlinear neutral dynamic equations

$$
[x(t)+p(t) x(g(t))]^{\Delta}+f(t, x(h(t)))=0
$$

on a time scale $\mathbb{T}$. Then Gao and Wang [7], Deng and Wang [6] discussed second-order nonlinear neutral dynamic equations

$$
\left[r(t)(x(t)+p(t) x(g(t)))^{\Delta}\right]^{\Delta}+f(t, x(h(t)))=0
$$

under different conditions successively. Inspired by [6, 7], Qiu [9] considered third-order nonlinear neutral dynamic equations

$$
\left(r_{1}(t)\left(r_{2}(t)(x(t)+p(t) x(g(t)))^{\Delta}\right)^{\Delta}\right)^{\Delta}+f(t, x(h(t)))=0
$$

under the condition $g(t) \leq t$, and established the existence of nonoscillatory solutions of equation (1). However, the conditions ensuring the existence of the nonoscillatory solutions tending to zero as $t \rightarrow \infty$ of equation (1) were exceptional, and as a result, the applications were within limits (see [9], Theorems 3.5 and 3.6).

(c) 2016 Qiu. This article is distributed under the terms of the Creative Commons Attribution 4.0 International License (http://creativecommons.org/licenses/by/4.0/), which permits unrestricted use, distribution, and reproduction in any medium, provided you give appropriate credit to the original author(s) and the source, provide a link to the Creative Commons license, and indicate if changes were made. 
In 2011, Mojsej and Tartal'ová [8] studied the asymptotic behavior of nonoscillatory solutions of third-order nonlinear differential equations with quasiderivatives of the form

$$
\left(\frac{1}{p(t)}\left(\frac{1}{r(t)} x^{\prime}(t)\right)^{\prime}\right)^{\prime}+q(t) f(x(t))=0, \quad t \geq a,
$$

and stated the necessary and sufficient conditions ensuring the existence of nonoscillatory solutions tending to zero as $t \rightarrow \infty$.

In this paper, we investigate the existence of nonoscillatory solutions tending to zero as $t \rightarrow \infty$ of equation (1) on a time scale $\mathbb{T}$, which satisfies inf $\mathbb{T}=t_{0}$ and $\sup \mathbb{T}=\infty$. We state the following conditions, which hold throughout this paper:

(C1) $r_{1}, r_{2} \in C_{r d}(\mathbb{T},(0, \infty))$.

(C2) $p \in C_{r d}(\mathbb{T},[0, \infty))$ and there exists a constant $p_{0}$ with $0 \leq p_{0}<1$ such that $\lim _{t \rightarrow \infty} p(t)=p_{0}$.

(C3) $g, h \in C_{r d}(\mathbb{T}, \mathbb{T}), g(t) \geq t$, and $\lim _{t \rightarrow \infty} h(t)=\infty$.

(C4) $f \in C(\mathbb{T} \times \mathbb{R}, \mathbb{R})$ and $x f(t, x)>0$ for $t \in \mathbb{T}$ and $x \neq 0$.

In the sequel, there are two cases of the function $f$ to be considered:

(C4A) There exist $q \in C(\mathbb{T},(0, \infty))$ and $f_{0} \in C(\mathbb{R}, \mathbb{R})$ such that $x f(t, x) \leq x q(t) f_{0}(x)$.

(C4B) $f(t, x)$ is nondecreasing in $x$.

Motivated by [8,9], we will establish the existence of nonoscillatory solutions tending to a zero of equation (1) by employing Kranoselskii's fixed point theorem and, finally, two examples are given to show the versatility of the conclusions.

Definition 1.1 A solution $x$ of equation (1) is said to be eventually positive (or eventually negative) if there exists $c \in \mathbb{T}$ such that $x(t)>0$ (or $x(t)<0)$ for all $t \geq c$ in $\mathbb{T}$. $x$ is said to be nonoscillatory if it is either eventually positive or eventually negative; otherwise, it is oscillatory.

\section{Auxiliary results}

Let $B C\left(\left[T_{0}, \infty\right)_{\mathbb{T}}, \mathbb{R}\right)$ denote the Banach space of all bounded continuous functions on $\left[T_{0}, \infty\right)_{\mathbb{T}}$ with the norm

$$
\|x\|=\sup _{t \in\left[T_{0}, \infty\right)_{\mathbb{T}}}|x(t)| .
$$

Definition 2.1 Let $X \subseteq B C\left[T_{0}, \infty\right)_{\mathbb{T}}$, we say that $X$ is uniformly Cauchy, if for any given $\epsilon>0$, there exists a $T_{1} \in\left[T_{0}, \infty\right)_{\mathbb{T}}$ such that $\left|x\left(t_{1}\right)-x\left(t_{2}\right)\right|<\epsilon$ for any $x \in X$ and $t_{1}, t_{2} \in$ $\left[T_{1}, \infty\right)_{\mathbb{T}}$.

Definition 2.2 $X$ is said to be equi-continuous on $[a, b]_{\mathbb{T}}$, if for any given $\epsilon>0$, there exists $\delta>0$ such that $\left|x\left(t_{1}\right)-x\left(t_{2}\right)\right|<\epsilon$ for any $x \in X$ and $t_{1}, t_{2} \in[a, b]_{\mathbb{T}}$ with $\left|t_{1}-t_{2}\right|<\delta$.

Lemma 2.3 ([10], Lemma 4) Suppose that $X \subseteq B C\left[T_{0}, \infty\right)_{\mathbb{T}}$ is bounded and uniformly Cauchy. Further, suppose that $X$ is equi-continuous on $\left[T_{0}, T_{1}\right]_{\mathbb{T}}$ for any $T_{1} \in\left[T_{0}, \infty\right)_{\mathbb{T}}$. Then $X$ is relatively compact.

Lemma 2.4 (Kranoselskii's fixed point theorem, see [5]) Suppose that $X$ is a Banach space and $\Omega$ is a bounded, convex, and closed subset of $X$. Suppose further that there exist two operators $U, S: \Omega \rightarrow X$ such that 
(1) $U x+$ Sy $\in \Omega$ for all $x, y \in \Omega$;

(2) $U$ is a contraction mapping;

(3) $S$ is completely continuous.

Then $U+S$ has a fixed point in $\Omega$.

Without loss of generality, we shall consider the case that eventually positive solutions of equation (1) in the following. It is because if $x(t)$ is an eventually negative solution of equation (1), then $y(t)=-x(t)$ will satisfy

$$
\left(r_{1}(t)\left(r_{2}(t)(y(t)+p(t) y(g(t)))^{\Delta}\right)^{\Delta}\right)^{\Delta}-f(t,-y(h(t)))=0 .
$$

Note that $\bar{f}(t, u):=-f(t,-u)$ satisfies (C4), and (C4A) or (C4B) similarly as $f(t, u)$.

Define

$$
z(t):=x(t)+p(t) x(g(t))
$$

then we will have the following lemma.

Lemma 2.5 Suppose that $x(t)$ is an eventually positive solution of equation (1), and there exists a constant $a \geq 0$ such that $\lim _{t \rightarrow \infty} z(t)=a$. Then we have

$$
\lim _{t \rightarrow \infty} x(t)=\frac{a}{1+p_{0}} .
$$

Proof Suppose that $x(t)$ is an eventually positive solution of equation (1). In view of (C3), there exists $t_{1} \in\left[t_{0}, \infty\right)_{\mathbb{T}}$ such that $x(t)>0, x(g(t))>0$ for $t \in\left[t_{1}, \infty\right)_{\mathbb{T}}$. We claim that $x(t)$ is bounded on $\left[t_{1}, \infty\right)_{\mathbb{T}}$. Assume not; then we have

$$
z(t)=x(t)+p(t) x(g(t)) \geq x(t) \rightarrow \infty
$$

which contradicts the fact that $\lim _{t \rightarrow \infty} z(t)=a$. Therefore, $x(t)$ is bounded. Then assume that

$$
\limsup _{t \rightarrow \infty} x(t)=\bar{x} \quad \text { and } \quad \liminf _{t \rightarrow \infty} x(t)=\underline{x} .
$$

Since $0 \leq p_{0}<1$, we have

$$
a \geq \bar{x}+p_{0} \underline{x} \text { and } a \leq \underline{x}+p_{0} \bar{x}
$$

which implies that $\bar{x} \leq \underline{x}$. So $\bar{x}=\underline{x}$, and we see that $\lim _{t \rightarrow \infty} x(t)$ exists and $\lim _{t \rightarrow \infty} x(t)=$ $a /\left(1+p_{0}\right)$. The proof is complete.

\section{Main results}

In this section, we state and prove our existence criteria for eventually positive solutions tending to zero as $t \rightarrow \infty$ of equation (1). 
Theorem 3.1 Assume that the function $f$ satisfies $(\mathrm{C} 4 \mathrm{~A})$ and there exists $M_{1}>0$ such that

$$
\int_{t_{0}}^{\infty} \frac{1}{r_{2}(t)} \Delta t=M_{1}<\infty
$$

and

$$
\int_{t_{0}}^{\infty} \int_{t_{0}}^{s} \frac{q(u)}{r_{1}(s)} \Delta u \Delta s<\infty
$$

Define $H_{1}(t)=\int_{t}^{\infty} \frac{1}{r_{2}(v)} \Delta v$, which satisfies $\lim _{t \rightarrow \infty} \frac{H_{1}(g(t))}{H_{1}(t)}=\eta_{1} \in(0,1]$. If there exists $L>0$ such that

$$
\left|f\left(t, x_{1}\right)-f\left(t, x_{2}\right)\right| \leq L \cdot q(t)\left|x_{1}-x_{2}\right|, \quad x_{1}, x_{2} \in\left[0,2 M_{1}\right],
$$

then equation (1) has an eventually positive solution $x(t)$ with $\lim _{t \rightarrow \infty} x(t)=0$, and there exists $T_{1} \in\left[t_{0}, \infty\right)_{\mathbb{T}}$ such that

$$
r_{2}(t) z^{\Delta}(t)<0, \quad r_{1}(t)\left(r_{2}(t) z^{\Delta}(t)\right)^{\Delta}<0, \quad t \in\left[T_{1}, \infty\right)_{\mathbb{T}} .
$$

Proof From (C2), for $0 \leq p_{0}<1$, choose $p_{1}$ such that $p_{0}<p_{1}<\left(1+4 p_{0}\right) / 5<1$. By (4) and (5), there exists $T_{0} \in\left[t_{0}, \infty\right)_{\mathbb{T}}$ such that

$$
\frac{5 p_{1}-1}{4} \leq p(t) \leq p_{1}<1, \quad p(t) \frac{H_{1}(g(t))}{H_{1}(t)} \geq \frac{5 p_{1}-1}{4} \eta_{1}, \quad t \in\left[T_{0}, \infty\right)_{\mathbb{T}},
$$

and

$$
\int_{T_{0}}^{\infty} \int_{T_{0}}^{s} \frac{q(u)}{r_{1}(s)} \Delta u \Delta s \leq \min \left\{\frac{1-p_{1} \eta_{1}}{4 K}, 1\right\}
$$

where $K=\max \left\{\left|f_{0}(x)\right|: x \in\left[0,2 M_{1}\right]\right\}$. From $(\mathrm{C} 3)$, there always exists $T_{1} \in\left(T_{0}, \infty\right)_{\mathbb{T}}$ such that

$$
h(t) \geq T_{0}, \quad t \in\left[T_{1}, \infty\right)_{\mathbb{T}} .
$$

Define

$$
\Omega_{1}=\left\{x(t) \in B C\left[T_{0}, \infty\right)_{\mathbb{T}}: H_{1}(t) \leq x(t) \leq 2 H_{1}(t)\right\} .
$$

It is clear that $\Omega_{1}$ is a bounded, convex, and closed subset of $B C\left[T_{0}, \infty\right)_{\mathbb{T}}$. For any $x \in \Omega_{1}$, by $(\mathrm{C} 4 \mathrm{~A})$ we have

$$
0<f(t, x(h(t))) \leq q(t) f_{0}(x(h(t))), \quad t \in\left[T_{1}, \infty\right)_{\mathbb{T}} .
$$

Define the operators $U_{1}$ and $S_{1}: \Omega_{1} \rightarrow B C\left[T_{0}, \infty\right)_{\mathbb{T}}$ as

$$
\left(U_{1} x\right)(t)=\frac{3}{2} p_{1} \eta_{1} H_{1}(t)-p(t) x(g(t)), \quad t \in\left[T_{0}, \infty\right)_{\mathbb{T}},
$$


and

$$
\left(S_{1} x\right)(t)= \begin{cases}\frac{3}{2} H_{1}(t)+\int_{t}^{\infty} \int_{T_{1}}^{v} \int_{T_{1}}^{s} \frac{f(u, x(h(u)))}{r_{1}(s) r_{2}(v)} \Delta u \Delta s \Delta v, & t \in\left[T_{1}, \infty\right)_{\mathbb{T}}, \\ \left(S_{1} x\right)\left(T_{1}\right), & t \in\left[T_{0}, T_{1}\right]_{\mathbb{T}} .\end{cases}
$$

Then we prove that $U_{1}$ and $S_{1}$ satisfy all the conditions in Lemma 2.4.

(1) For any $x, y \in \Omega_{1}$ and $t \in\left[T_{1}, \infty\right)_{\mathbb{T}}$, by (7)-(9) we obtain

$$
\begin{aligned}
& \left(U_{1} x\right)(t)+\left(S_{1} y\right)(t) \\
& \leq \frac{3\left(1+p_{1} \eta_{1}\right)}{2} H_{1}(t)-\frac{5 p_{1}-1}{4} \eta_{1} H_{1}(t)+\int_{t}^{\infty} \int_{T_{1}}^{v} \int_{T_{1}}^{s} \frac{q(u) f_{0}(y(h(u)))}{r_{1}(s) r_{2}(v)} \Delta u \Delta s \Delta v \\
& \leq \frac{3\left(1+p_{1} \eta_{1}\right)}{2} H_{1}(t)-\frac{5 p_{1}-1}{4} \eta_{1} H_{1}(t)+K H_{1}(t) \int_{T_{1}}^{\infty} \int_{T_{1}}^{s} \frac{q(u)}{r_{1}(s)} \Delta u \Delta s \\
& \leq \frac{3\left(1+p_{1} \eta_{1}\right)}{2} H_{1}(t)-\frac{5 p_{1}-1}{4} \eta_{1} H_{1}(t)+\frac{1-p_{1} \eta_{1}}{4} H_{1}(t) \\
& =\frac{7+\eta_{1}}{4} H_{1}(t) \leq 2 H_{1}(t)
\end{aligned}
$$

and

$$
\begin{aligned}
& \left(U_{1} x\right)(t)+\left(S_{1} y\right)(t) \\
& =\frac{3\left(1+p_{1} \eta_{1}\right)}{2} H_{1}(t)-p(t) x(g(t))+\int_{t}^{\infty} \int_{T_{1}}^{v} \int_{T_{1}}^{s} \frac{f(u, y(h(u)))}{r_{1}(s) r_{2}(v)} \Delta u \Delta s \Delta v \\
& \geq \frac{3\left(1+p_{1} \eta_{1}\right)}{2} H_{1}(t)-2 p_{1} \eta_{1} H_{1}(t)=\frac{3-p_{1} \eta_{1}}{2} H_{1}(t)>H_{1}(t) .
\end{aligned}
$$

Similarly, $H_{1}(t) \leq\left(U_{1} x\right)(t)+\left(S_{1} y\right)(t) \leq 2 H_{1}(t)$ also holds for any $x, y \in \Omega_{1}$ and $t \in$ $\left[T_{0}, T_{1}\right]_{\mathbb{T}}$. It follows that $U_{1} x+S_{1} y \in \Omega_{1}$ for any $x, y \in \Omega_{1}$.

(2) For any $x, y \in \Omega_{1}$ and $t \in\left[T_{0}, \infty\right)_{\mathbb{T}}$, we always have

$$
\left|\left(U_{1} x\right)(t)-\left(U_{1} y\right)(t)\right|=|p(t)(x(g(t))-y(g(t)))| \leq p_{1} \sup _{\left.t \in\left[T_{0}, \infty\right)\right)_{\mathbb{T}}}|x(t)-y(t)| .
$$

It follows that $\left\|U_{1} x-U_{1} y\right\| \leq p_{1}\|x-y\|$ for any $x, y \in \Omega_{1}$. That is, $U_{1}$ is a contraction mapping.

(3) For $t \in\left[T_{0}, \infty\right)_{\mathbb{T}}$, we have

$$
\left(S_{1} x\right)(t) \leq \frac{3}{2} H_{1}(t)+\frac{1-p_{1} \eta_{1}}{4} H_{1}(t)=\frac{7-p_{1} \eta_{1}}{4} H_{1}(t)<2 H_{1}(t)
$$

and

$$
\left(S_{1} x\right)(t)>\frac{3}{2} H_{1}(t)>H_{1}(t)
$$

It is easy to see that $S_{1}$ maps $\Omega_{1}$ into $\Omega_{1}$.

For any $x \in \Omega_{1}$ and $t \in\left[T_{0}, \infty\right)_{\mathbb{T}}$, let $x_{n} \in \Omega_{1}$ and $\left\|x_{n}-x\right\| \rightarrow 0$ as $n \rightarrow \infty$. For $t \in$ $\left[T_{1}, \infty\right)_{\mathbb{T}}$, by $(4),(6)$, and $(8)$, we have 


$$
\begin{aligned}
& \left|\left(S_{1} x_{n}\right)(t)-\left(S_{1} x\right)(t)\right| \\
& \quad \leq \int_{t}^{\infty} \int_{T_{1}}^{v} \int_{T_{1}}^{s} \frac{\left|f\left(u, x_{n}(h(u))\right)-f(u, x(h(u)))\right|}{r_{1}(s) r_{2}(v)} \Delta u \Delta s \Delta v \\
& \quad \leq L \int_{T_{1}}^{\infty} \frac{1}{r_{2}(v)} \Delta v \cdot \int_{T_{1}}^{\infty} \int_{T_{1}}^{s} \frac{q(u)\left|x_{n}(h(u))-x(h(u))\right|}{r_{1}(s)} \Delta u \Delta s \\
& \quad \leq L M_{1} \cdot \sup _{t \in\left[T_{0}, \infty\right)_{\mathbb{T}}}\left|x_{n}(t)-x(t)\right| .
\end{aligned}
$$

For $t \in\left[T_{0}, T_{1}\right]_{\mathbb{T}}$, we also have the result above. By Lebesgue's dominated convergence theorem (see Chapter 5 in [11]), it follows that

$$
\left\|S_{1} x_{n}-S_{1} x\right\| \rightarrow 0, \quad n \rightarrow \infty .
$$

Therefore, $S_{1}$ is continuous.

It is obvious that $S_{1} \Omega_{1}$ is bounded. On the other hand, by (4), for any $\epsilon>0$ there exists $T_{2} \in\left[T_{1}, \infty\right)_{\mathbb{T}}$ such that

$$
H_{1}\left(T_{2}\right)=\int_{T_{2}}^{\infty} \frac{1}{r_{2}(t)} \Delta t<\frac{\epsilon}{2 K+3}
$$

For $t_{1}, t_{2} \in\left[T_{0}, T_{1}\right]_{\mathbb{T}}$, we always have $\left|\left(S_{1} x\right)\left(t_{1}\right)-\left(S_{1} x\right)\left(t_{2}\right)\right|=0$. Then, for any $x \in \Omega_{1}$ and $t_{1}, t_{2} \in\left[T_{2}, \infty\right)_{\mathbb{T}}$, we have

$$
\begin{aligned}
& \left|\left(S_{1} x\right)\left(t_{1}\right)-\left(S_{1} x\right)\left(t_{2}\right)\right| \\
& \leq\left|\int_{t_{1}}^{\infty} \int_{T_{1}}^{v} \int_{T_{1}}^{s} \frac{f(u, x(h(u)))}{r_{1}(s) r_{2}(v)} \Delta u \Delta s \Delta v-\int_{t_{2}}^{\infty} \int_{T_{1}}^{v} \int_{T_{1}}^{s} \frac{f(u, x(h(u)))}{r_{1}(s) r_{2}(v)} \Delta u \Delta s \Delta v\right| \\
& \quad+\frac{3}{2}\left|H_{1}\left(t_{1}\right)-H_{1}\left(t_{2}\right)\right| \\
& \quad \leq 2 \int_{T_{2}}^{\infty} \int_{T_{1}}^{v} \int_{T_{1}}^{s} \frac{f(u, x(h(u)))}{r_{1}(s) r_{2}(v)} \Delta u \Delta s \Delta v+3 H_{1}\left(T_{2}\right) \\
& \leq 2 K H_{1}\left(T_{2}\right) \int_{T_{1}}^{\infty} \int_{T_{1}}^{s} \frac{q(u)}{r_{1}(s)} \Delta u \Delta s+3 H_{1}\left(T_{2}\right) \\
& \leq(2 K+3) H_{1}\left(T_{2}\right)<\epsilon .
\end{aligned}
$$

It is clear that $S_{1} \Omega_{1}$ is uniformly Cauchy.

For $x \in \Omega_{1}$ and $t_{1}, t_{2} \in\left[\min \left\{T_{1}-1, T_{0}\right\}, T_{2}+1\right]_{\mathbb{T}}$, we have

$$
\begin{aligned}
& \left|\left(S_{1} x\right)\left(t_{1}\right)-\left(S_{1} x\right)\left(t_{2}\right)\right| \\
& \leq\left|\int_{t_{1}}^{t_{2}} \int_{T_{1}}^{v} \int_{T_{1}}^{s} \frac{f(u, x(h(u)))}{r_{1}(s) r_{2}(v)} \Delta u \Delta s \Delta v\right|+\frac{3}{2}\left|\int_{t_{1}}^{t_{2}} \frac{1}{r_{2}(t)} \Delta t\right| \\
& \quad \leq K \int_{T_{1}}^{\infty} \int_{T_{1}}^{s} \frac{q(u)}{r_{1}(s)} \Delta u \Delta s\left|\int_{t_{1}}^{t_{2}} \frac{1}{r_{2}(t)} \Delta t\right|+\frac{3}{2}\left|\int_{t_{1}}^{t_{2}} \frac{1}{r_{2}(t)} \Delta t\right| \\
& \quad \leq\left(K+\frac{3}{2}\right)\left|H_{1}\left(t_{1}\right)-H_{1}\left(t_{2}\right)\right| .
\end{aligned}
$$


Therefore, there exists $0<\delta<1$ such that $\left|\left(S_{1} x\right)\left(t_{1}\right)-\left(S_{1} x\right)\left(t_{2}\right)\right|<\epsilon$ for any $t_{1}, t_{2} \in\left[T_{0}\right.$, $\left.T_{2}+1\right]_{\mathbb{T}}$ with $\left|t_{1}-t_{2}\right|<\delta$. We conclude that $S_{1} \Omega_{1}$ is equi-continuous.

According to Lemma 2.3, it follows that $S_{1} \Omega_{1}$ is relatively compact and $S_{1}$ is completely continuous. Then by Lemma 2.4, there exists $x \in \Omega_{1}$ such that $\left(U_{1}+S_{1}\right) x=x$. It means that $x(t)$ is an eventually positive solution of equation (1), and for $t \in\left[T_{1}, \infty\right)_{\mathbb{T}}$, we have

$$
x(t)=\frac{3\left(1+p_{1} \eta_{1}\right)}{2} H_{1}(t)-p(t) x(g(t))+\int_{t}^{\infty} \int_{T_{1}}^{v} \int_{T_{1}}^{s} \frac{f(u, x(h(u)))}{r_{1}(s) r_{2}(v)} \Delta u \Delta s \Delta v .
$$

As

$$
\int_{t}^{\infty} \int_{T_{1}}^{v} \int_{T_{1}}^{s} \frac{f(u, x(h(u)))}{r_{1}(s) r_{2}(v)} \Delta u \Delta s \Delta v \leq K H_{1}(t) \int_{T_{1}}^{\infty} \int_{T_{1}}^{s} \frac{q(u)}{r_{1}(s)} \Delta u \Delta s
$$

for $t \in\left[T_{1}, \infty\right)_{\mathbb{T}}$, and

$$
\lim _{t \rightarrow \infty} K H_{1}(t) \int_{T_{1}}^{\infty} \int_{T_{1}}^{s} \frac{q(u)}{r_{1}(s)} \Delta u \Delta s=0
$$

it follows that $\lim _{t \rightarrow \infty} z(t)=0$. By Lemma 2.5, we obtain $\lim _{t \rightarrow \infty} x(t)=0$. Furthermore, for $t \in\left[T_{1}, \infty\right)_{\mathbb{T}}$, we have

$$
r_{2}(t) z^{\Delta}(t)=-\frac{3\left(1+p_{1} \eta_{1}\right)}{2}-\int_{T_{1}}^{t} \int_{T_{1}}^{s} \frac{f(u, x(h(u)))}{r_{1}(s)} \Delta u \Delta s<0
$$

and

$$
r_{1}(t)\left(r_{2}(t) z^{\Delta}(t)\right)^{\Delta}=-\int_{T_{1}}^{t} f(u, x(h(u))) \Delta u<0
$$

The proof is complete.

Theorem 3.2 Assume that

$$
\int_{t_{0}}^{\infty} \frac{1}{r_{2}(t)} \Delta t=\infty \quad \text { or } \quad \int_{t_{0}}^{\infty} \int_{t_{0}}^{v} \frac{1}{r_{1}(s) r_{2}(v)} \Delta s \Delta v=\infty
$$

Then equation (1) has no eventually positive solution $x(t)$, for which $r_{2}(t) z^{\Delta}(t)$ and $r_{1}(t)\left(r_{2}(t) z^{\Delta}(t)\right)^{\Delta}$ are both eventually negative.

Proof Suppose that $x(t)$ is an eventually positive solution of equation (1), and there exists $T_{0} \in\left[t_{0}, \infty\right)_{\mathbb{T}}$ such that

$$
r_{2}(t) z^{\Delta}(t)<0, \quad r_{1}(t)\left(r_{2}(t) z^{\Delta}(t)\right)^{\Delta}<0, \quad t \in\left[T_{0}, \infty\right)_{\mathbb{T}} .
$$

By (C3), there exists $T_{1} \in\left(T_{0}, \infty\right)_{\mathbb{T}}$ such that $h(t) \geq T_{0}$ for $t \in\left[T_{1}, \infty\right)_{\mathbb{T}}$. Integrating (1) from $T_{1}$ to $s \in\left[\sigma\left(T_{1}\right), \infty\right)_{\mathbb{T}}$, by $(\mathrm{C} 4)$ we obtain

$$
r_{1}(s)\left(r_{2}(s) z^{\Delta}(s)\right)^{\Delta}-r_{1}\left(T_{1}\right)\left(r_{2}\left(T_{1}\right) z^{\Delta}\left(T_{1}\right)\right)^{\Delta}=-\int_{T_{1}}^{s} f(u, x(h(u))) \Delta u<0,
$$


which implies that

$$
\left(r_{2}(s) z^{\Delta}(s)\right)^{\Delta}<\frac{r_{1}\left(T_{1}\right)\left(r_{2}\left(T_{1}\right) z^{\Delta}\left(T_{1}\right)\right)^{\Delta}}{r_{1}(s)} .
$$

Integrating (11) from $T_{1}$ to $v \in\left[\sigma\left(T_{1}\right), \infty\right)_{\mathbb{T}}$, we obtain

$$
r_{2}(v) z^{\Delta}(v)-r_{2}\left(T_{1}\right) z^{\Delta}\left(T_{1}\right)<r_{1}\left(T_{1}\right)\left(r_{2}\left(T_{1}\right) z^{\Delta}\left(T_{1}\right)\right)^{\Delta} \int_{T_{1}}^{v} \frac{1}{r_{1}(s)} \Delta s
$$

or

$$
z^{\Delta}(v)<\frac{r_{2}\left(T_{1}\right) z^{\Delta}\left(T_{1}\right)}{r_{2}(v)}+\frac{r_{1}\left(T_{1}\right)\left(r_{2}\left(T_{1}\right) z^{\Delta}\left(T_{1}\right)\right)^{\Delta}}{r_{2}(v)} \int_{T_{1}}^{v} \frac{1}{r_{1}(s)} \Delta s .
$$

Integrating (12) from $T_{1}$ to $t \in\left[\sigma\left(T_{1}\right), \infty\right)_{\mathbb{T}}$, we obtain

$$
\begin{aligned}
z(t)< & z\left(T_{1}\right)+r_{2}\left(T_{1}\right) z^{\Delta}\left(T_{1}\right) \int_{T_{1}}^{t} \frac{1}{r_{2}(v)} \Delta v \\
& +r_{1}\left(T_{1}\right)\left(r_{2}\left(T_{1}\right) z^{\Delta}\left(T_{1}\right)\right)^{\Delta} \int_{T_{1}}^{t} \int_{T_{1}}^{v} \frac{1}{r_{1}(s) r_{2}(v)} \Delta s \Delta v .
\end{aligned}
$$

Letting $t \rightarrow \infty$, we have $z(t) \rightarrow-\infty$. It is a contradiction because $z(t)=x(t)+p(t) x(g(t))$ is eventually positive. The proof is complete.

By Theorems 3.1 and 3.2, we have the following corollary.

Corollary 3.3 Assume that

$$
\int_{t_{0}}^{\infty} \int_{t_{0}}^{s} \frac{q(u)}{r_{1}(s)} \Delta u \Delta s<\infty
$$

If the function $f$ satisfies $(\mathrm{C} 4 \mathrm{~A})$ and there exists $L>0$ such that

$$
\left|f\left(t, x_{1}\right)-f\left(t, x_{2}\right)\right| \leq L \cdot q(t)\left|x_{1}-x_{2}\right|, \quad x_{1}, x_{2} \in\left[0,2 \int_{t_{0}}^{\infty} \frac{1}{r_{2}(t)} \Delta t\right],
$$

then $\int_{t_{0}}^{\infty} \frac{1}{r_{2}(t)} \Delta t<\infty$ is a necessary and sufficient condition for equation (1) to have an eventually positive solution $x(t)$ satisfying the requirement that $\lim _{t \rightarrow \infty} x(t)=0$ and $r_{2}(t) z^{\Delta}(t)$, $r_{1}(t)\left(r_{2}(t) z^{\Delta}(t)\right)^{\Delta}$ are both eventually negative.

Theorem 3.4 Assume that the function $f$ satisfies (C4A) and there exists $M_{2}>0$ such that

$$
\int_{t_{0}}^{\infty} \int_{v}^{\infty} \frac{1}{r_{1}(s) r_{2}(v)} \Delta s \Delta v=M_{2}<\infty
$$

and

$$
\int_{t_{0}}^{\infty} q(t) \Delta t<\infty
$$


Define $H_{2}(t)=\int_{t}^{\infty} \int_{v}^{\infty} \frac{1}{r_{1}(s) r_{2}(v)} \Delta s \Delta v$, which satisfies $\lim _{t \rightarrow \infty} \frac{H_{2}(g(t))}{H_{2}(t)}=\eta_{2} \in(0,1]$. If there exists $L>0$ such that

$$
\left|f\left(t, x_{1}\right)-f\left(t, x_{2}\right)\right| \leq L \cdot q(t)\left|x_{1}-x_{2}\right|, \quad x_{1}, x_{2} \in\left[0,2 M_{2}\right]
$$

then equation (1) has an eventually positive solution $x(t)$ with $\lim _{t \rightarrow \infty} x(t)=0$, and there exists $T_{1} \in\left[t_{0}, \infty\right)_{\mathbb{T}}$ such that

$$
r_{2}(t) z^{\Delta}(t)<0, \quad r_{1}(t)\left(r_{2}(t) z^{\Delta}(t)\right)^{\Delta}>0, \quad t \in\left[T_{1}, \infty\right)_{\mathbb{T}} .
$$

Proof From (C2), for $0 \leq p_{0}<1$, choose $p_{1}$ such that $p_{0}<p_{1}<\left(1+4 p_{0}\right) / 5<1$. By (13) and (14), there exists $T_{0} \in\left[t_{0}, \infty\right)_{\mathbb{T}}$ such that

$$
\frac{5 p_{1}-1}{4} \leq p(t) \leq p_{1}<1, \quad p(t) \frac{H_{2}(g(t))}{H_{2}(t)} \geq \frac{5 p_{1}-1}{4} \eta_{2}, \quad t \in\left[T_{0}, \infty\right)_{\mathbb{T}},
$$

and

$$
\int_{T_{0}}^{\infty} q(t) \Delta t \leq \min \left\{\frac{1-p_{1} \eta_{2}}{4 K}, 1\right\}
$$

where $K=\max \left\{\left|f_{0}(x)\right|: x \in\left[0,2 M_{2}\right]\right\}$. Similarly, there always exists $T_{1} \in\left(T_{0}, \infty\right)_{\mathbb{T}}$ such that $h(t) \geq T_{0}$ for $t \in\left[T_{1}, \infty\right)_{\mathbb{T}}$.

Define

$$
\Omega_{2}=\left\{x(t) \in B C\left[T_{0}, \infty\right)_{\mathbb{T}}: H_{2}(t) \leq x(t) \leq 2 H_{2}(t)\right\} .
$$

Then $\Omega_{2}$ is also a bounded, convex, and closed subset of $B C\left[T_{0}, \infty\right)_{\mathbb{T}}$. For any $x \in \Omega_{2}$, by (C4A) we have

$$
0<f(t, x(h(t))) \leq q(t) f_{0}(x(h(t))), \quad t \in\left[T_{1}, \infty\right)_{\mathbb{T}} .
$$

Define the operators $U_{2}$ and $S_{2}: \Omega_{2} \rightarrow B C\left[T_{0}, \infty\right)_{\mathbb{T}}$ as

$$
\left(U_{2} x\right)(t)=\frac{3}{2} p_{1} \eta_{2} H_{2}(t)-p(t) x(g(t)), \quad t \in\left[T_{0}, \infty\right)_{\mathbb{T}},
$$

and

$$
\left(S_{2} x\right)(t)= \begin{cases}\frac{3}{2} H_{2}(t)+\int_{t}^{\infty} \int_{v}^{\infty} \int_{s}^{\infty} \frac{f(u, x(h(u)))}{r_{1}(s) r_{2}(v)} \Delta u \Delta s \Delta v, & t \in\left[T_{1}, \infty\right)_{\mathbb{T}} \\ \left(S_{2} x\right)\left(T_{1}\right), & t \in\left[T_{0}, T_{1}\right]_{\mathbb{T}} .\end{cases}
$$

Similarly to the proof of Theorem 3.1, we can conclude that $U_{2}$ and $S_{2}$ satisfy all the conditions in Lemma 2.4. Therefore, there exists $x \in \Omega_{2}$ such that $\left(U_{2}+S_{2}\right) x=x$, which means that $x(t)$ is an eventually positive solution of equation (1). It follows that for $t \in$ $\left[T_{1}, \infty\right)_{\mathbb{T}}$, we have

$$
x(t)=\frac{3\left(1+p_{1} \eta_{2}\right)}{2} H_{2}(t)-p(t) x(g(t))+\int_{t}^{\infty} \int_{v}^{\infty} \int_{s}^{\infty} \frac{f(u, x(h(u)))}{r_{1}(s) r_{2}(v)} \Delta u \Delta s \Delta v .
$$


As

$$
\int_{t}^{\infty} \int_{v}^{\infty} \int_{s}^{\infty} \frac{f(u, x(h(u)))}{r_{1}(s) r_{2}(v)} \Delta u \Delta s \Delta v \leq K H_{2}(t) \int_{T_{1}}^{\infty} q(u) \Delta u
$$

for $t \in\left[T_{1}, \infty\right)_{\mathbb{T}}$, and

$$
\lim _{t \rightarrow \infty} K H_{2}(t) \int_{T_{1}}^{\infty} q(u) \Delta u=0
$$

we have $\lim _{t \rightarrow \infty} z(t)=0$, which implies that $\lim _{t \rightarrow \infty} x(t)=0$ by Lemma 2.5. For $t \in$ $\left[T_{1}, \infty\right)_{\mathbb{T}}$, we obtain

$$
r_{2}(t) z^{\Delta}(t)=-\frac{3\left(1+p_{1} \eta_{2}\right)}{2} \int_{t}^{\infty} \frac{1}{r_{1}(s)} \Delta s-\int_{t}^{\infty} \int_{s}^{\infty} \frac{f(u, x(h(u)))}{r_{1}(s)} \Delta u \Delta s<0
$$

and

$$
r_{1}(t)\left(r_{2}(t) z^{\Delta}(t)\right)^{\Delta}=\frac{3\left(1+p_{1} \eta_{2}\right)}{2}+\int_{t}^{\infty} f(u, x(h(u))) \Delta u>0
$$

The proof is complete.

Remark 3.5 Actually, (6) in Theorem 3.1 and (15) in Theorem 3.4 will hold especially when $f(t, x)=q(t) f_{0}(x)$ and $f_{0}(x)$ satisfies the Lipschitz condition on $\left[0,2 M_{1}\right]$ and $\left[0,2 M_{2}\right]$, respectively.

Remark 3.6 Letting $\mathbb{T}=[a, \infty), r_{1}(t)=\frac{1}{p(t)}, r_{2}(t)=\frac{1}{r(t)}, p(t)=0$, and the function $f$ satisfying (C4A), equation (1) is simplified as (2). Therefore, Theorems 3.1, 3.2, 3.4, and Corollary 3.3 complement and extend the results in Mojsej and Tartal'ová [8].

In the sequel, we change the condition of the function $f$ from $(\mathrm{C} 4 \mathrm{~A})$ to $(\mathrm{C} 4 \mathrm{~B})$, and get some similar conclusions as follows.

Theorem 3.7 Assume that the function $f$ satisfies (C4B) and

$$
\int_{t_{0}}^{\infty} \frac{1}{r_{2}(t)} \Delta t<\infty
$$

and

$$
\int_{t_{0}}^{\infty} \int_{t_{0}}^{s} \frac{f\left(u, 2 H_{1}(h(u))\right)}{r_{1}(s)} \Delta u \Delta s<\infty
$$

where $H_{1}(t)=\int_{t}^{\infty} \frac{1}{r_{2}(v)} \Delta v$, which satisfies $\lim _{t \rightarrow \infty} \frac{H_{1}(g(t))}{H_{1}(t)}=\eta_{1} \in(0,1]$. Then equation (1) has an eventually positive solution $x(t)$ with $\lim _{t \rightarrow \infty} x(t)=0$, and there exists $T_{1} \in\left[t_{0}, \infty\right)_{\mathbb{T}}$ such that

$$
r_{2}(t) z^{\Delta}(t)<0, \quad r_{1}(t)\left(r_{2}(t) z^{\Delta}(t)\right)^{\Delta}<0, \quad t \in\left[T_{1}, \infty\right)_{\mathbb{T}} .
$$


Proof From (C2), for $0 \leq p_{0}<1$, choose $p_{1}$ such that $p_{0}<p_{1}<\left(1+4 p_{0}\right) / 5<1$. By (17) and (18), there exists $T_{0} \in\left[t_{0}, \infty\right)_{\mathbb{T}}$ such that (7) holds and

$$
\int_{T_{0}}^{\infty} \int_{T_{0}}^{s} \frac{f\left(u, 2 H_{1}(h(u))\right)}{r_{1}(s)} \Delta u \Delta s \leq \min \left\{\frac{1-p_{1} \eta_{1}}{4}, 1\right\} .
$$

Similarly, there always exists $T_{1} \in\left(T_{0}, \infty\right)_{\mathbb{T}}$ such that $h(t) \geq T_{0}$ for $t \in\left[T_{1}, \infty\right)_{\mathbb{T}}$.

Define $\Omega_{1}$ as in Theorem 3.1. For any $x \in \Omega_{1}$, by (C4B) we have

$$
0<f(t, x(h(t))) \leq f\left(t, 2 H_{1}(h(t))\right), \quad t \in\left[T_{1}, \infty\right)_{\mathbb{T}} .
$$

In addition, define $U_{1}$ and $S_{1}$ as in Theorem 3.1. It is obvious that $U_{1}$ and $S_{1}$ also satisfy all the conditions in Lemma 2.4. Hence, there exists $x \in \Omega_{1}$ such that $\left(U_{1}+S_{1}\right) x=x$. That is, $x(t)$ is an eventually positive solution of equation (1). For $t \in\left[T_{1}, \infty\right)_{\mathbb{T}}$, it follows that

$$
x(t)=\frac{3\left(1+p_{1} \eta_{1}\right)}{2} H_{1}(t)-p(t) x(g(t))+\int_{t}^{\infty} \int_{T_{1}}^{v} \int_{T_{1}}^{s} \frac{f(u, x(h(u)))}{r_{1}(s) r_{2}(v)} \Delta u \Delta s \Delta v .
$$

As

$$
\int_{t}^{\infty} \int_{T_{1}}^{v} \int_{T_{1}}^{s} \frac{f(u, x(h(u)))}{r_{1}(s) r_{2}(v)} \Delta u \Delta s \Delta v \leq H_{1}(t) \int_{T_{1}}^{\infty} \int_{T_{1}}^{s} \frac{f\left(u, 2 H_{1}(h(u))\right)}{r_{1}(s)} \Delta u \Delta s
$$

for $t \in\left[T_{1}, \infty\right)_{\mathbb{T}}$, and

$$
\lim _{t \rightarrow \infty} H_{1}(t) \int_{T_{1}}^{\infty} \int_{T_{1}}^{s} \frac{f\left(u, 2 H_{1}(h(u))\right)}{r_{1}(s)} \Delta u \Delta s=0,
$$

it follows that $\lim _{t \rightarrow \infty} z(t)=0$. By Lemma 2.5, we obtain $\lim _{t \rightarrow \infty} x(t)=0$. Furthermore, for $t \in\left[T_{1}, \infty\right)_{\mathbb{T}}$, it satisfies

$$
r_{2}(t) z^{\Delta}(t)<0, \quad r_{1}(t)\left(r_{2}(t) z^{\Delta}(t)\right)^{\Delta}<0, \quad t \in\left[T_{1}, \infty\right)_{\mathbb{T}} .
$$

The proof is complete.

Theorem 3.8 Assume that the function $f$ satisfies (C4.B) and

$$
\int_{t_{0}}^{\infty} \int_{v}^{\infty} \frac{1}{r_{1}(s) r_{2}(v)} \Delta s \Delta v<\infty
$$

and

$$
\int_{t_{0}}^{\infty} f\left(t, 2 H_{2}(h(t))\right) \Delta t<\infty
$$

where $H_{2}(t)=\int_{t}^{\infty} \int_{v}^{\infty} \frac{1}{r_{1}(s) r_{2}(v)} \Delta s \Delta v$, which satisfies $\lim _{t \rightarrow \infty} \frac{H_{2}(g(t))}{H_{2}(t)}=\eta_{2} \in(0,1]$. Then equation (1) has an eventually positive solution $x(t)$ with $\lim _{t \rightarrow \infty} x(t)=0$, and there exists $T_{1} \in\left[t_{0}, \infty\right)_{\mathbb{T}}$ such that

$$
r_{2}(t) z^{\Delta}(t)<0, \quad r_{1}(t)\left(r_{2}(t) z^{\Delta}(t)\right)^{\Delta}>0, \quad t \in\left[T_{1}, \infty\right)_{\mathbb{T}} .
$$


Proof From (C2), for $0 \leq p_{0}<1$, choose $p_{1}$ such that $p_{0}<p_{1}<\left(1+4 p_{0}\right) / 5<1$. By (19) and (20), there exists $T_{0} \in\left[t_{0}, \infty\right)_{\mathbb{T}}$ such that (16) holds and

$$
\int_{T_{0}}^{\infty} f\left(t, 2 H_{2}(h(t))\right) \Delta t \leq \min \left\{\frac{1-p_{1} \eta_{2}}{4}, 1\right\}
$$

From (C3), there always exists $T_{1} \in\left(T_{0}, \infty\right)_{\mathbb{T}}$ such that $h(t) \geq T_{0}$ for $t \in\left[T_{1}, \infty\right)_{\mathbb{T}}$.

Define $\Omega_{2}$ as in Theorem 3.4. For any $x \in \Omega_{1}$, by (C4B) we have

$$
0<f(t, x(h(t))) \leq f\left(t, 2 H_{2}(h(t))\right), \quad t \in\left[T_{1}, \infty\right)_{\mathbb{T}} .
$$

In addition, define the operators $U_{2}$ and $S_{2}$ as in Theorem 3.4. It is obvious that $U_{2}$ and $S_{2}$ also satisfy all the conditions in Lemma 2.4. Then there exists $x \in \Omega_{2}$ such that $\left(U_{2}+S_{2}\right) x=$ $x$, which means that $x(t)$ is an eventually positive solution of equation (1). For $t \in\left[T_{1}, \infty\right)_{\mathbb{T}}$, it follows that

$$
x(t)=\frac{3\left(1+p_{1} \eta_{2}\right)}{2} H_{2}(t)-p(t) x(g(t))+\int_{t}^{\infty} \int_{v}^{\infty} \int_{s}^{\infty} \frac{f(u, x(h(u)))}{r_{1}(s) r_{2}(v)} \Delta u \Delta s \Delta v .
$$

As

$$
\int_{t}^{\infty} \int_{v}^{\infty} \int_{s}^{\infty} \frac{f(u, x(h(u)))}{r_{1}(s) r_{2}(v)} \Delta u \Delta s \Delta v \leq H_{2}(t) \int_{T_{1}}^{\infty} f\left(u, 2 H_{2}(h(u))\right) \Delta u
$$

for $t \in\left[T_{1}, \infty\right)_{\mathbb{T}}$, and

$$
\lim _{t \rightarrow \infty} H_{2}(t) \int_{T_{1}}^{\infty} f\left(u, 2 H_{2}(h(u))\right) \Delta u=0
$$

we have $\lim _{t \rightarrow \infty} z(t)=0$, which implies that $\lim _{t \rightarrow \infty} x(t)=0$ by Lemma 2.5. We also obtain

$$
r_{2}(t) z^{\Delta}(t)<0, \quad r_{1}(t)\left(r_{2}(t) z^{\Delta}(t)\right)^{\Delta}>0, \quad t \in\left[T_{1}, \infty\right)_{\mathbb{T}} .
$$

The proof is complete.

Remark 3.9 Indeed, if the function $f$ satisfies both of (C4A) and (C4B), compared to Theorems 3.1 and 3.4, Theorems 3.7 and 3.8 are more convenient to employ, respectively.

\section{Examples}

In this section, we give two examples to show the application of our results. The first example is presented to illustrate Theorems 3.1 and 3.7.

Example 4.1 Let $\mathbb{T}=\bigcup_{n=1}^{\infty}[2 n-1,2 n]$. Consider

$$
\left(t^{5}\left(t^{2}\left(x(t)+\frac{t-1}{2 t} x(t+2)\right)^{\Delta}\right)^{\Delta}\right)^{\Delta}+t^{2} x(t)+\frac{x^{3}(t)}{t}=0
$$

where $r_{1}(t)=t^{5}, r_{2}(t)=t^{2}, p(t)=\frac{t-1}{2 t}, g(t)=t+2, h(t)=t, f(t, x)=t^{2} x+\frac{x^{3}}{t}, t_{0}=1$. 
Take $q(t)=t^{2}$ and $f_{0}(x)=x+x^{3}$. It is obvious that the coefficients of equation (21) satisfy (C1)-(C4), (C4A), and (C4B). Since

$$
\begin{aligned}
& \int_{t_{0}}^{\infty} \frac{1}{r_{2}(t)} \Delta t=\int_{1}^{\infty} \frac{1}{t^{2}} \Delta t=M_{1}<2, \\
& H_{1}(t)=\int_{t}^{\infty} \frac{1}{r_{2}(v)} \Delta v=\int_{t}^{\infty} \frac{1}{v^{2}} \Delta v<2, \\
& \lim _{t \rightarrow \infty} \frac{H_{1}(g(t))}{H_{1}(t)}=\lim _{t \rightarrow \infty} \frac{\int_{t+2}^{\infty} \frac{1}{v^{2}} \Delta v}{\int_{t}^{\infty} \frac{1}{v^{2}} \Delta v}=\lim _{t \rightarrow \infty} \frac{t^{2}}{(t+2)^{2}}=1, \\
& \int_{t_{0}}^{\infty} \int_{t_{0}}^{s} \frac{q(u)}{r_{1}(s)} \Delta u \Delta s=\int_{1}^{\infty} \int_{1}^{s} \frac{u^{2}}{s^{5}} \Delta u \Delta s<\int_{1}^{\infty} \int_{1}^{s} \frac{1}{s^{3}} \Delta u \Delta s<\int_{1}^{\infty} \frac{1}{s^{2}} \Delta s<\infty,
\end{aligned}
$$

and, for any $x_{1}, x_{2} \in[0,4]$,

$$
\begin{aligned}
\left|f\left(t, x_{1}\right)-f\left(t, x_{2}\right)\right| & =\left|t^{2}\left(x_{1}-x_{2}\right)+\frac{1}{t}\left(x_{1}^{3}-x_{2}^{3}\right)\right| \\
& \leq t^{2}\left(\left|x_{1}-x_{2}\right|+\left|x_{1}^{3}-x_{2}^{3}\right|\right) \leq\left(1+x_{1}^{2}+x_{1} x_{2}+x_{2}^{2}\right) \cdot t^{2}\left|x_{1}-x_{2}\right| \\
& \leq 49 \cdot q(t)\left|x_{1}-x_{2}\right|,
\end{aligned}
$$

by Theorem 3.1 we see that equation (21) has an eventually positive solution $x(t)$ with $\lim _{t \rightarrow \infty} x(t)=0$, and $r_{2}(t) z^{\Delta}(t), r_{1}(t)\left(r_{2}(t) z^{\Delta}(t)\right)^{\Delta}$ are both eventually negative.

On the other hand, since $H_{1}(t)<2$, we obtain

$$
f\left(u, 2 H_{1}(h(u))\right)=u^{2} \cdot 2 H_{1}(u)+\frac{8 H_{1}^{3}(u)}{u} \leq u^{2}\left(2 H_{1}(u)+8 H_{1}^{3}(u)\right)<68 u^{2}
$$

and

$$
\begin{aligned}
\int_{t_{0}}^{\infty} \int_{t_{0}}^{s} \frac{f\left(u, 2 H_{1}(h(u))\right)}{r_{1}(s)} \Delta u \Delta s & <68 \int_{1}^{\infty} \int_{1}^{s} \frac{u^{2}}{s^{5}} \Delta u \Delta s \\
& <68 \int_{1}^{\infty} \int_{1}^{s} \frac{1}{s^{3}} \Delta u \Delta s<68 \int_{1}^{\infty} \frac{1}{s^{2}} \Delta s<\infty
\end{aligned}
$$

By Theorem 3.7 we have the same conclusion.

Then the second example demonstrates Theorems 3.4 and 3.8 .

Example 4.2 Let $\mathbb{T}=\bigcup_{n=1}^{\infty}\left[2^{n}-1,2^{n}\right]$. Consider

$$
\left(t^{2}\left(t\left(\left(1+\frac{1}{t}\right) x(t)\right)^{\Delta}\right)^{\Delta}\right)^{\Delta}+\frac{x\left(\frac{t}{2}\right)}{t^{2}}=0
$$

where $r_{1}(t)=t^{2}, r_{2}(t)=t, p(t)=\frac{1}{t}, g(t)=t, h(t)=\frac{t}{2}, f(t, x)=\frac{x}{t^{2}}, t_{0}=1$. 
Take $q(t)=\frac{1}{t^{2}}$ and $f_{0}(x)=x$. It is obvious that the coefficients of equation (22) satisfy (C1)-(C4), (C4A), and (C4B). Since

$$
\begin{aligned}
& \int_{t_{0}}^{\infty} \int_{v}^{\infty} \frac{1}{r_{1}(s) r_{2}(v)} \Delta s \Delta v=\int_{1}^{\infty} \int_{v}^{\infty} \frac{1}{s^{2} v} \Delta s \Delta v \\
& =\int_{1}^{\infty} \int_{1}^{s} \frac{1}{s^{2} v} \Delta v \Delta s=M_{2}<\infty, \\
& H_{2}(t)=\int_{t}^{\infty} \int_{v}^{\infty} \frac{1}{r_{1}(s) r_{2}(v)} \Delta s \Delta v=\int_{t}^{\infty} \int_{v}^{\infty} \frac{1}{s^{2} v} \Delta s \Delta v \leq M_{2} \text {, } \\
& \lim _{t \rightarrow \infty} \frac{H_{2}(g(t))}{H_{2}(t)}=\lim _{t \rightarrow \infty} \frac{H_{2}(t)}{H_{2}(t)}=1, \\
& \int_{t_{0}}^{\infty} q(t) \Delta t=\int_{1}^{\infty} \frac{1}{t^{2}} \Delta t<\infty
\end{aligned}
$$

and for any $x_{1}, x_{2} \in\left[0,2 M_{2}\right]$,

$$
\left|f\left(t, x_{1}\right)-f\left(t, x_{2}\right)\right|=\frac{\left|x_{1}-x_{2}\right|}{t^{2}}=1 \cdot q(t)\left|x_{1}-x_{2}\right|,
$$

by Theorem 3.4 we see that equation (22) has an eventually positive solution $x(t)$ with $\lim _{t \rightarrow \infty} x(t)=0, r_{2}(t) z^{\Delta}(t)$ is eventually negative and $r_{1}(t)\left(r_{2}(t) z^{\Delta}(t)\right)^{\Delta}$ is eventually positive.

On the other hand, since $H_{2}(t) \leq M_{2}$, we obtain

$$
f\left(t, 2 H_{2}(h(t))\right)=\frac{2 H_{2}\left(\frac{t}{2}\right)}{t^{2}} \leq \frac{2 M_{2}}{t^{2}}
$$

and

$$
\int_{t_{0}}^{\infty} f\left(t, 2 H_{2}(h(t))\right) \Delta t \leq 2 M_{2} \int_{1}^{\infty} \frac{1}{t^{2}} \Delta t<\infty .
$$

By Theorem 3.8 we have the same conclusion.

\section{Competing interests}

The author declares that he has no competing interests.

\section{Acknowledgements}

This project was supported by the NNSF of China (no. 11271379), the Program of Cultivation for Outstanding Young

Scholars Sponsored by Guangdong Province (ZX03240302) and the Program of Cultivation for Young Scholars Sponsored by Shunde Polytechnic (2015-KJZX080).

Received: 6 October 2015 Accepted: 4 January 2016 Published online: 13 January 2016

\section{References}

1. Hilger, S: Ein Maßkettenkalkül mit Anwendung auf Zentrumsmannigfaltigkeiten. Ph.D. thesis, Universität Würzburg (1988)

2. Hilger, S: Analysis on measure chain - a unified approach to continuous and discrete calculus. Results Math. 18, 18-56 (1990)

3. Agarwal, RP, Bohner, M: Basic calculus on time scales and some of its applications. Results Math. 35, 3-22 (1999)

4. Agarwal, RP, Bohner, M, O'Regan, D, Peterson, A: Dynamic equations on time scales: a survey. J. Comput. Appl. Math. $141,1-26$ (2002)

5. Chen, YS: Existence of nonoscillatory solutions of $n$th order neutral delay differential equations. Funkc. Ekvacioj 35 , 557-570 (1992)

6. Deng, XH, Wang, QR: Nonoscillatory solutions to second-order neutral functional dynamic equations on time scales. Commun. Appl. Anal. 18, 261-280 (2014) 
7. Gao, J, Wang, QR: Existence of nonoscillatory solutions to second-order nonlinear neutral dynamic equations on time scales. Rocky Mt. J. Math. 43, 1521-1535 (2013)

8. Mojsej, I, Tartal'ová, A: On nonoscillatory solutions tending to zero of third-order nonlinear differential equations. Tatra Mt. Math. Publ. 48, 135-143 (2011)

9. Qiu, YC: Nonoscillatory solutions to third-order neutral dynamic equations on time scales. Adv. Differ. Equ. 2014, 309 (2014)

10. Zhu, ZQ, Wang, QR: Existence of nonoscillatory solutions to neutral dynamic equations on time scales. J. Math. Anal. Appl. 335, 751-762 (2007)

11. Bohner, M, Peterson, A: Dynamic Equations on Time Scales: An Introduction with Applications. Birkhäuser, Boston (2001)

Submit your manuscript to a SpringerOpen ${ }^{\odot}$ journal and benefit from:

- Convenient online submission

Rigorous peer review

- Immediate publication on acceptance

- Open access: articles freely available online

- High visibility within the field

- Retaining the copyright to your article 